\title{
Una experiencia en el laboratorio de ciencias para el alumnado del grado de Educación Infantil
}

\author{
Dulsat Ortiz, Carles \\ Departamento de Pedagoxía e Didáctica, Facultade de Ciencias da Educación.
}

\section{RESUMEN}

La experiencia que se describe parte de los resultados iniciales del alumnado de esta materia y su relación con las ciencias naturales. Se identificó al inicio del curso cual era la relación del alumnado con las ciencias de la naturaleza para al final del curso académico averiguar cómo, desde una propuesta más participativa, se mejora ese grado de aceptación de los conceptos científicos y su interés por las ciencias de la naturaleza. La propuesta en el laboratorio fue pedir a cada uno de los pequeños grupos de trabajo la exposición de tres experimentos científicos junto a la información científica que explica cada uno de ellos. Esta propuesta considera entre otras cuestiones los intereses del alumnado en una materia que para muchos de ellos resulta compleja en la comprensión de sus conceptos. El buscar la información, el preparar, explicar y exponer los experimentos hace que algunos de esos conceptos se acerquen a su comprensión. Esta propuesta contribuye a la reflexión crítica en la búsqueda de información y en la aplicación de conceptos una vez iniciados en los mecanismos con las que aproximarse a las ciencias naturales.

PALABRAS CLAVE: ciencias de la naturaleza, educación infantil, aprendizaje activo. 


\section{CITA RECOMENDADA:}

Dulsat Ortiz, C. (2019): Una experiencia en el laboratorio de ciencias para el alumnado del grado de Educación Infantil. En De la Torre Fernández, E. (ed.) (2019). Contextos universitarios transformadores: construíndo espazos de aprendizaxe. III Xornadas de Innovación Docente. Cufie. Universidade da Coruña. A Coruña (pág. 213-228).

DOl capítulo: https://doi.org/10.17979/spudc.9788497497121.213

DOl libro: https://doi.org/10.17979/spudc.9788497497121

\section{ABSTRACT}

The experience described is based on the initial results of the students of this subject and their relationship with the natural sciences. It was identified at the beginning of the course which was the relation of the students with the natural sciences for the end of the academic year to find out how, from a more participative proposal, that degree of acceptance of the scientific concepts and their interest in the sciences of nature. The proposal in the laboratory was to ask each of the small working groups to present three scientific experiments together with the scientific information that each of them explains. This proposal considers among other issues the interests of students in a subject that for many of them is complex in understanding their concepts. The search for information, preparing, explaining and exposing the experiments makes some of these concepts closer to their understanding. This proposal contributes to critical reflection in the search for information and in the application of concepts once initiated in the mechanisms with which to approach the natural sciences.

KEY WORDS: nature sciences, early childhood education, active learning, 


\section{INTRODUCCIÓN.}

Varios años impartiendo la materia Ensino das Ciencias da Natureza en el grado de Educación Infantil en la Facultad de Ciencias de la Educación, me hicieron ver las dificultadas que la mayoría de los estudiantes tenían ante los conceptos de ciencias que en su mayoría se presentaban en el laboratorio. La metodología usada hasta el momento era expositiva y totalmente dirigida, donde el alumnado repetía los experimentos y escuchaba la teoría científica que la acompañaba. Al reflexionar sobre esto, se ha intentado mejorar los aprendizajes del alumnado y, para ello, se elaboró un cuestionario ad hoc al inicio de curso para conocer intereses e inquietudes acerca de las ciencias naturales, y otro cuestionario al final del curso para averiguar cómo sus expectativas se habían visto alcanzadas, haciendo especial hincapié en las sesiones en el laboratorio y el trabajo que debían desarrollar para llevarlo a cabo.

Este cambio en la estrategia metodológica busca además una mejora en la calidad, ya no sólo de la enseñanza, sino que también en el aprendizaje. Por ello, en el siguiente apartado se describirá de forma concreta la experiencia lleva a cabo durante el pasado curso 2017-2018. Se aleja la experiencia que aquí se presenta de los métodos pasivos centrados en la memorización y en la rutina y repetición de los experimentos para llevarlos lo más cerca posible a métodos más activos donde el alumnado es protagonista de su propio aprendizaje $y$, para ello, se parte de sus intereses como ya planteaba Decroly (1987) en sus centros de interés, así como partir de los conocimientos iniciales del propio alumnado (Ausubel et al., 1983) y construir nuevos conocimientos asentados en estos iniciales.

A este aporte pedagógico y psicológico, cabe añadir las aportaciones de Chevallard (1991). Este autor introduce el concepto de transposición didáctica o cómo conseguir que el saber científico se convierta en un saber apropiado para ser enseñado, intentando conseguir un estadio intermedio entre las explicaciones eruditas y las explicaciones cotidianas. Esta transposición didáctica está conformada por dos momentos donde la transposición del objeto a enseñar pasa por un estadio externo cuando el saber científico debe ser enseñado; y, una 
transposición interna cuando el saber enseñado ha sido adquirido por el alumnado y donde interviene el profesor de forma directa. Por lo que el maestro se convierte en el que adapta y acerca los conocimientos científicos al aula.

Este conocimiento científico es mucho más complejo y con un mayor grado de construcción que el pensamiento cotidiano (Chalmers, 1992; y, Chalmers, 1999). Además, para el pensamiento científico, son necesarias una serie de habilidades cognitivas mediante las cuales se construye desde la percepción de la realidad que nos permite conocerla. Es el saber científico una forma evolucionada del pensamiento cotidiano, y con este saber científico se acerca el conocimiento de los objetos de la realidad independientemente de quien observa. Lo destacable del pensamiento científico es la íntima relación con habilidades cognitivas como el lenguaje y la capacidad de hacerse preguntas, de reflexionar, acerca de aquello que tenemos a nuestro alrededor.

Cabe superar la propuesta constructivista ante el inicio de la adquisición del conocimiento científico ya que consideran las ideas previas que explican la realidad como erróneos. Para ello, los estudios de Chi \& Roscoe (2002) proponen que las ideas previas que se construyen en las primeras edades son mecanismos cognitivos que facilitaran posteriormente la construcción de aprendizajes que expliquen la realidad de forma más fehaciente. De estos mecanismos cognitivos diferencian dos tipos: los preconceptos o conocimientos inocentes que son fácilmente sustituibles y revisados por la futura formación; y los errores conceptuales 0 ideas erróneas arraigadas en la mente de las personas, que con la edad sea hace cada vez más difícil su sustitución. Son ideas previas o intuitivas que dan una explicación de la realidad a partir del conocimiento que se tiene en ese momento y que contribuye a interpretar los fenómenos y los objetos que suceden alrededor y que permite dar explicación del comportamiento.

Por todo esto, los objetivos que pretenden esta experiencia fueron: primero, acercar la ciencia de forma más participativa al alumnado; segundo, mostrar las consecuencias en el alumnado 
de la metodología usada durante las prácticas; y, tercero, conseguir un mayor conocimiento de las ciencias de la naturaleza desde un cambio metodológico.

\section{DESCRIPCIÓN DE LA EXPERIENCIA}

Esta experiencia tiene lugar durante las horas destinadas a prácticas en el laboratorio como elemento de aprendizaje para la materia de Ensino de Ciencias da Natureza perteneciente al grado de educación infantil en la facultad de ciencias de la educación de la Universidade da Coruña. Durante ese tiempo de prácticas en un espacio tan concreto, el grupo de más de sesenta alumnas se divide en tres grupos de poco más de 20 alumnas, bajo el nombre de grupos interactivos (a partir de ahora Gl), que, a su vez, se dividen en varios subgrupos de 40 5 (a partir de ahora $P G$ ) o pequeños grupos de trabajo.

Al inicio de curso, se explica al gran grupo el funcionamiento de las prácticas. A continuación, detallamos de forma cronológica tanto las acciones a realizar por el alumnado como las que debe realizar el docente. Primero, durante las siguientes semanas previas al inicio de las prácticas, se deben enviar los experimentos a través de la plataforma Moodle. Segundo, dos semanas antes al inicio de las prácticas, el docente comprobará dentro de cada GI la no coincidencia de los experimentos entre los PG, la correcta redacción de los mismos y la correspondencia con la teoría que debe acompañar el experimento. En caso de coincidencia, se reunirá con los grupos afectados y se consensuarán los cambios oportunos. Además, se pedirán voluntarios para iniciar las sesiones de prácticas. Tercero, se acompañará al PG responsable de la sesión práctica al laboratorio para enseñarle y orientarle en la preparación del material necesario. Esto se realizará el día antes de la sesión, abordando también la normativa de uso del laboratorio, la preparación del material y las adaptaciones 0 modificaciones a realizar en el experimento para su correcta ejecución. Esta segunda función del docente es importante porque en años anteriores el alumnado asistía al laboratorio para solo repetir los experimentos y anotar aquellos conceptos que los acompañaban rellenando una ficha que se les entregaba, además de tutorizar al grupo en las dudas que le podrían surgir 
y de qué manera acercar los experimentos al resto de compañeras y na acertada explicación del concepto científico de cada uno de ellos. Y cuarto, el día de la sesión, el grupo tutor explica los experimentos y la teoría relacionada siendo el papel del profesor acompañar a ese grupo y contribuir a una explicación más adecuada, si procede de la teoría que explican del experimento. El resto de grupos deben resolver una serie de preguntas elaboradas para el conocimiento previo y posterior del experimento que se va a llevar a cabo. También, es el grupo encargado de recoger y limpiar el material y el laboratorio para que quede tal y como se encontró antes de empezar. Al finalizar, las sesiones de prácticas se realizan una evaluación conjunta de la experiencia realizada tanto desde una coevaluación entre los miembros del PG como una heteroevaluación bidireccional alumnado y docente.

\section{RESULTADOS}

\subsection{MUESTRA.}

La muestra de esta experiencia fue el alumnado del grupo A de segundo de educación infantil de la materia de Ensino das Ciencias a Natureza. Es una población de 65 alumnas de las cuales 61 fueron la muestra generadora de datos porque dos alumnas matriculadas no se presentaron en todo el curso y otras dos decidieron realizar la modalidad no presencial que permite la normativa de evaluación en la Facultade de Ciencias da Educación de la Universidade da Coruña.

\subsection{INSTRUMENTOS.}

Se usaron dos instrumentos construidos ad hoc para valorar y evaluar esta experiencia. El primero está compuesto por 7 ítems y se presenta al inicio de la materia; el segundo, de 11 ítems, se presenta al final de la última sesión de prácticas. Estos dos cuestionarios contaban con un elemento de codificación mediante el cual se pretende mantener el anonimato de las participantes y de las respuestas dadas. Estos cuestionarios fueron rellenados en el aula y de forma presencial. 


\subsection{RECOPILACIÓN DE DATOS.}

El análisis cuantitativo se realizó mediante el programa estadístico SPSS-22 para las pruebas descriptivas y para las pruebas paramétricas, mientras que el análisis cualitativo se realizó con el software QSR NVIV0-10.

\subsection{RESULTADOS CUANTITATIVOS.}

Destaca que el rango de edad de las alumnas se encuentra entre los 19 a los 30 años, siendo el $50 \%$ menores de 21. La categoría sexo no se incluyó en el estudio porque en el grupo de 65 únicamente 6 eran chicos. En la imagen 1, se presentan los porcentajes de las titulaciones con las que el alumnado ha accedido a los estudios de educación infantil, y como estos estudios previos pueden condicionar la aceptación a las ciencias naturales por el tiempo que llevan sin actualizar los conocimientos en este campo. En las imágenes 2 y 3 , podemos observar los grados de aceptación inicial y final en relación a las ciencias naturales respecto a las vivencias de la metodología usada. Esta metodología ha permitido aumentar la aceptación en el alumnado respecto a las ciencias naturales y, además, ha conseguido no disminuir la aceptación de ninguna de las alumnas. De los resultados obtenidos, cabe destacar que el $78,3 \%$ de las alumnas se dedican exclusivamente a los estudios y el resto, 21,7\%, además de estudiar están trabajando bien a tiempo completo o bien a tiempo parcial. 


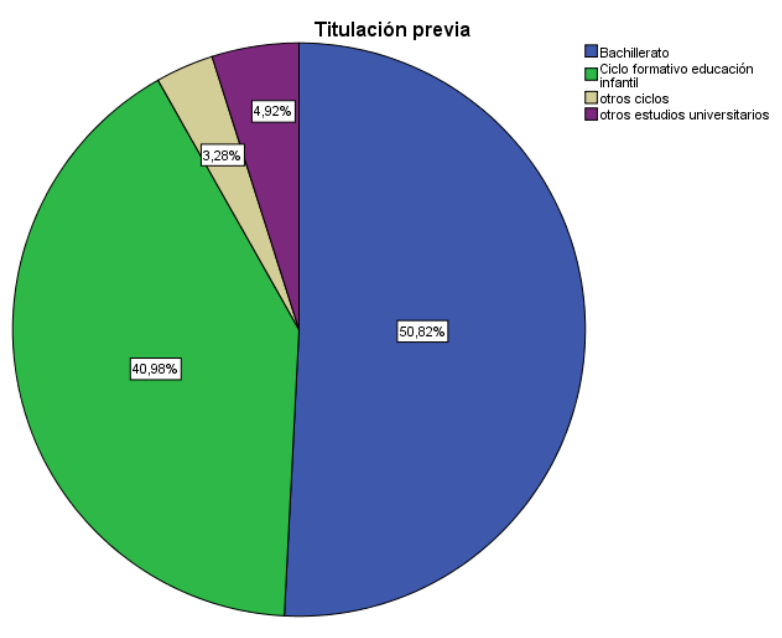

Imagen 1. Titulación de acceso a los estudios

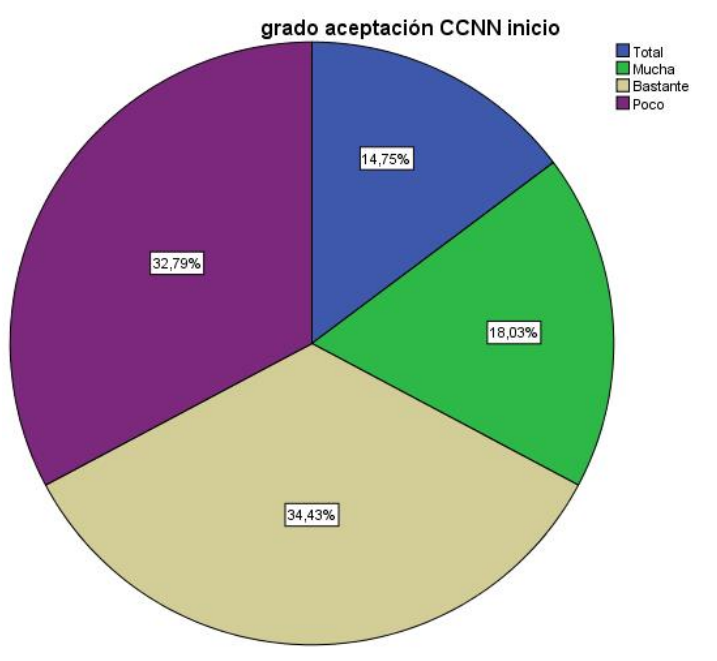

Imagen 2. Grado aceptación inicial ciencias naturales 


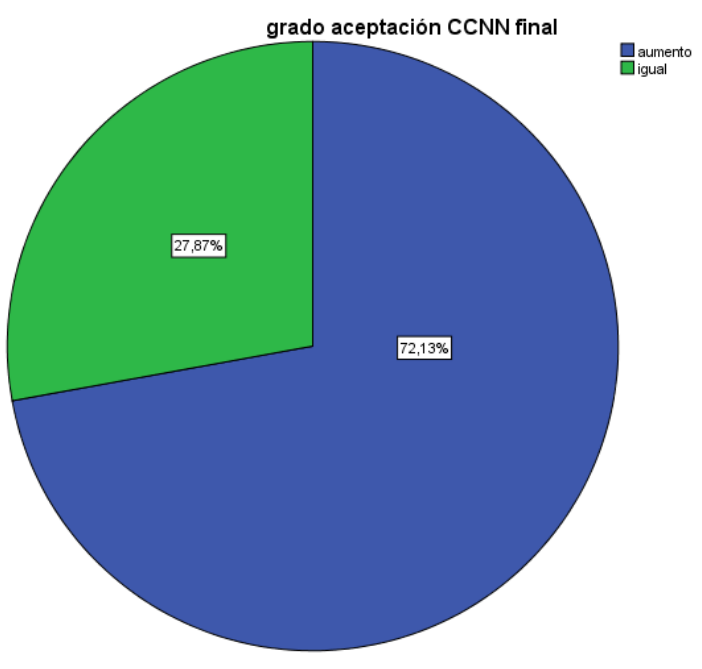

Imagen 3. Grado aceptación final ciencias naturales

En la tabla 1, constamos que, para los dos grados, inicial y final de aceptación de las ciencias naturales, existen diferencias significativas en las medias de ambos grupos, con lo que se rechaza la hipótesis nula de igual de las medias y aceptamos la hipótesis alternativa de medias diferentes, asumiendo el aumento en la aceptación de las ciencias naturales en la muestra a estudio.

\begin{tabular}{lc}
\hline \multicolumn{2}{c}{ Prueba T (muestras relacionadas) } \\
\hline & Sign. \\
\hline Grado acept. inicial & 0.000 \\
Grado acept. final & \\
\hline
\end{tabular}

Tabla 1. Significación grados de aceptación

Para dos muestras independientes, no existen diferencias significativas en la igualdad de las medias en la dedicación a los estudios del alumnado respecto a las variables independientes de titulación previa y de los dos grados de aceptación inicial y final, con lo que constatamos 
que la condición de trabajar y estudiar a la vez no representa diferencias significativas en estas tres variables en relación a aquellas alumnas que únicamente estudian.

La pertenencia a diferentes grupos interactivos no presenta diferencias significativas asumiendo la igualdad de las varianzas y, el formar parte de un grupo u otro, no representa diferencias significativas para los grados de aceptación inicial y final de las alumnas. Existen diferencias significativas entre las alumnas que trabajan y las que únicamente estudian respecto al grupo de edad, siendo mayor las diferencias de edad respecto la mediana en el grupo que trabaja que de aquellas que no trabajan donde la diferencia de edad se encuentra en un rango menor, cumpliéndose el supuesto de homocedasticidad.

\subsection{RESULTADOS CUALITATIVOS.}

El análisis cualitativo separa las respuestas del alumnado en función de las consideraciones que expresan en la pregunta referente al grado de mejora en el conocimiento de las ciencias gracias a la metodología usada en el laboratorio. Así para el grupo que considera que ha mejorado sean establecido cuatro grandes nódulos de información: nuevos conocimientos y mayor interés por las ciencias con un $31,8 \%$ de las respuestas, como por ejemplo lo que indican en sus respuestas: "me ha acercado a experimentos y explicaciones que no conocí, lo que ha sido positivo" (al. 5_a) y "Ios profesores anteriores que trabajan esta materia no 10 hacía de manera motivadora" (al. 17_a), respectivamente. Le siguen las respuestas que les ha resultado de utilidad como docentes con un $25 \%$ de las respuestas: "nos sirvió para el día de mañana saber cómo trabajar con los niños, lo relacionado con la naturaleza" (al. 9_a) por último, dentro de este grupo que han mejorado está la implicación en el día a día con un 11,4\%: "la veo que se puede aplicar en la vida cotidiana sin necesidad de rebuscar recursos" (al. 9_a).

En el grupo que consideran que sus conocimientos siguieron igual que se han construido tres grandes nódulos: Ya existía interés y conocimiento con un 70,5\% de las respuestas dentro de este grupo: "porque las sigo viendo igual de interesant4es que antes, solo que ahora de forma 
más experiencial" (al. 4_b). Le siguen las respuestas agrupadas dentro del nódulo grado previo de aceptación muy elevado con un 17,6\%: "porque tenía un alto grado de aceptación a la ciencia" (al. 3_b). El último grupo de respuestas están orientadas a las pocas horas dedicadas con un 11,8\%: "porque le dedicamos pocas horas" (al. 6_b).

Se pidió al alumnado tres dificultades con las que se encontraron durante las prácticas en el laboratorio. Las respuestas más comunes han sido: la organización de las sesiones con un $35,1 \%$, le sigue el exponer ante el resto de compañeros con un 16,4\%. Un 12,9\% consideran que no hubo dificultades, y le sigue el tiempo, la duración de las sesiones con un $10,6 \%$. No muy lejos se responde que la dificultad estuvo en el vocabulario científico con un $9,4 \%$ y el material disponible en el laboratorio con un $7,1 \%$, y el espacio limitado que supone el laboratorio con un $4,8 \%$. Cierran estas respuestas el horario de tarde con un $2,4 \%$ y que no fue útil para la vida cotidiana con un $1,3 \%$.

También se les preguntó por tres ventajas de esta metodología donde lo más respondido ha sido la adquisición de conocimientos con un 30,7\%. Le sigue de lejos: el carácter lúdico y entretenido con un 15,3\%, exploración y trabajo en grupo con un 14,6\%, y manipulación y experimentación con un 13,1\%. Al 10,2\% de las respuestas del alumnado les resultó interesante la metodología usada; y, el 7,3\% consideran que esta metodología da dinamismo a las sesiones. Cierran las respuestas tres nódulos menos representados: la guía del profesor con un 4,4\%, la mejora en la exposición con un 2,9\%, y la innovación que supone esta metodología con un $1,5 \%$.

\section{DISCUSIÓN}

Desde los datos cuantitativos, se puede considerar que ni la pertinencia a un grupo interactivo 0 el compartir estudios con otras obligaciones como el trabajo, no representa diferencias significativas en el alumnado. Recordando que la asistencia es obligatoria y que esta es la única materia que imparte docencia en horario de tarde. Respecto a esta organización de las sesiones, cabe hacer énfasis en la organización horaria donde todos los grupos pasan por las 
sesiones de primera hora de la mañana y por las sesiones de tarde de forma lo más paritaria posible. El alumnado que trabaja de tarde se les permitió asistir a otro grupo.

Respecto a la comparación entre los grados de aceptación inicial y final hacia las ciencias naturales, cabe destacar que hay diferencias significativas dirigidas hacia una mayor aceptación de esta disciplina y adquisición de sus conocimientos tal y como se refiere en los estudios de Gaete y Camacho (2017) en las aportaciones de nuevas pedagogías en el ámbito de la enseñanza de las ciencias y como la ciencia se aprende mejor desde prácticas, experiencias que sean significativas en el aprendizaje. Coincide con la aceptación e importancia que expresan el alumnado en el estudio de Cantó, Pro y Solbes (2016) con respecto a la importancia de la experimentación y experiencias en la enseñanza de las ciencias para educación infantil.

Dentro de las dificultadas mostradas por el alumnado, destaca por encima de las demás la poca organización de las sesiones. Esto se debe a una coincidencia de conceptos en experimentos distintos, porque se priorizó la coincidencia de los experimentos recibidos, pero no hubo previsión en la coincidencia de conceptos.

De las ventajas nombradas por el alumnado, destaca la adquisición de conocimientos, el aprendizaje significativo; aunque la mayoría de estas respuestas van siempre acompañadas por la motivación, el interés y/o el carácter lúdico y de entretenimiento de las sesiones. Coincide con las conclusiones de Busquets, Silva y Larrosa (2016) donde la enseñanza y aprendizaje de la química se facilitaría con más experimentación y salidas de campo, así como con López Rúa y Tamayo (2012) en su investigación acerca de las ventajas que suponer el aprendizaje en el laboratorio para biología y química. Las respuestas del alumnado especifican la mejora en la aceptación de las ciencias naturales, y se observa que la metodología usada en las sesiones del laboratorio son un factor que cabría profundizar en futuras investigaciones. Para ello, existen aproximaciones a metodologías activas donde el alumnado asume el protagonismo en las sesiones desde la relación de experiencias y experimentos hasta la 
resolución de problemas, superando la enseñanza transmitida y magistral donde se acercan a la memorización (Campanario \& Moya, 1999).

La segunda dificultad encontrada por el alumnado fue la exposición delante del resto de compañeras, el representar el rol docente. Esto puede coincidir con los estudios de Galagovsky, Bonán y Adúriz (1998) respecto a la poca percepción que tienen los docentes como comunicadores. Esta dificultad también ha sido vista como una ventaja a la hora de facilitar y mejorar la exposición y ser una primera oportunidad de ponerse ante el grupo. Implica acercarse a tareas y problemas por parte de aquellos que menos comprenden la materia. Además del escenario, el laboratorio supone la zona de interacción motivadora (Vygotsky, 1979).

\section{CONCLUSIONES}

Las conclusiones se agrupan en cuatro bloques: la organización de las prácticas, la metodología usada, las relaciones en el aula y los conocimientos adquiridos.

- Organización de las prácticas: Cabe concluir que, para los próximos cursos, la organización de las sesiones debe ser más ajustada, con menos repetición de conceptos científicos y manteniendo el número de estudiantes lo más igualado y constante posible. Algunas alumnas tienen dificultades para asistir en horario de tarde. Además, el trabajo en grupo es intenso y requiere a todas las participantes en todo momento. Los grupos con alumnas que trabajan deben estar bien identificados para, si es necesario, facilitar el cambio de grupo interactivo, siempre que se mantenga el número de alumnas en cada sesión.

- Metodología: Aún con las dificultades expresadas, ha resultado acertada, pues las respuestas lo destacan tanto por la adquisición de conocimientos como por la motivación durante los experimentos y la manipulación que ofrece. Consideramos que es una metodología que contribuye a una mayor participación del alumnado. Además, sienten como suyo aquello que están realizando y exponiendo delante de sus 
compañeras, y no como algo impuesto por el docente, que se repite de forma rutinaria, se responden a las preguntas planteadas, se entrega y se termina olvidando. La metodología usada ha sido unánimemente aceptada y considerada por el alumnado como adecuada para sus aprendizajes.

- Relaciones en el aula: Muchas de las reticencias del alumnado nacen en las relaciones en el aula, el ponerse ante sus compañeras y del poco respecto que consideran que reciben respecto a quienes las están escuchando. Desde la posición de docente, cabe hacer más hincapié en la atención y comprensión para con sus compañeras, pues todas serán en algún momento quienes conducirán la sesión.

- Conocimientos adquiridos: Es mayoritaria en el alumnado la percepción de que durante las prácticas ha adquirido nuevos conocimientos. Estos han sido desde una forma vivencial y significativa, pero pasaron muy desapercibida a lo largo de las sesiones los conceptos que autores como Chi \& Roscoe (2002) presentaban en sus estudios y el centro de interés de Decroly terminó transformándose en facilidad de acceso y búsqueda de información hacia los experimentos que debían exponer, alejándose en algunos casos de los intereses propios iniciales.

\section{REFERENCIAS}

Ausubel, D. P., Novak, J. D. \& Hanesian, H. (1983). Psicología educativa. Un punto de vista cognoscitivo. México: Trías editorial.

Busquets, T., Silva, M. \& Larrosa, P. (2016). Reflexiones sobre el aprendizaje de las ciencias naturales. Nuevas aproximaciones y desafíos. Estudios pedagógicos, número especial 40 años, 117-135.

Campanario, J. M. \& Moya, A. (1999). ¿Cómo enseñar ciencias? Principales tendencias y propuestas. Investigaciones didácticas, 17(2), pp. 179-192. 
Cantó, J., Pro, A. de \& Solbes, J. (2016). ¿Qué ciencias se enseñan y cómo se hace en las aulas de educación infantil? La visión de los maestros en formación inicial. Enseñanza de las ciencias, 34(3), pp. 25-50. http://dx.doi.org/10.5565/rev/ensciencias.1870 Chalmers, A. F. (1992). La ciencia y como se elabora. Madrid: Editorial Siglo XXI. Chalmers, A. F. (1999). ¿Qué es esa cosa Ilamada ciencia? Madrid: Editorial Siglo XXI. Chevallard, Y. (1991). La transposition didactique: du savoir savant au savoir enseigné. Grenoble: La Pensée Sauvage.

Chi, M. T. H., \& Roscoe, R. D. (2002). The processes and challenges of conceptual change. In Limon M., Mason L. (eds) Reconsidering conceptual change: Issues in theory and practice, pp. 3-27. Dordrecht: Springer. https://doi.org/10.1007/0-306-47637-1_1

Decroly, 0. (1987). Experiencias educativas e innovadoras. Madrid: editorial Passat.

Gaete, M. \& Camacho, J. (2017). Vivencias de practicantes de pedagogías en ciencias. Educaçao e Pesquisa, 43(2), pp. 341-356. http://dx.doi.org/10.1590/s15179702201609146976

López Rúa, A. M. \& Tamayo, 0. E. (2012). Las prácticas de laboratorio en la enseñanza de las ciencias naturales. Revista Latinoamericana de Estudios Educativos,8(1), pp.145-166.

Vygotsky, L. S. (1979). El desarrollo de los procesos psicológicos superiores. Barcelona: crítica. 
